\title{
Maxima - An Open Alternative for Engineering Education
}

\author{
doi:10.3991/ijep.v1i1.1598 \\ K. Žáková \\ Slovak University of Technology, Bratislava, Slovakia
}

\begin{abstract}
The aim of the article is to present a new possible way of an interactive teaching. The effort is to substitute proprietary software that is commonly used in educational process by open technologies. Since the subject of this article is oriented to the engineering area we devoted our attention to open software and we chose Maxima. Moreover, this tool can be available not only locally on the desktop computer or the notebook but also online via Internet. In the paper we also introduce examples of the implementation.
\end{abstract}

Index Terms - computer aided engineering, control design, online services, student experiments.

\section{INTRODUCTION}

In the last decade the development of the low cost software (free and open source software) takes increasingly bigger and bigger importance. It brings competition to the proprietary software products including open source platforms.

We decided to devote our attention to open computer algebra systems that can be used in education of technical topics. We could choose from several possibilities, e.g. Axiom, Maxima, SymPy, Sage, Yacas, etc. After comparison of developed functionality, operating system support, software ongoing support (year of the last stable version) we decided to use Maxima software environment.

Our aim was to increase interactivity of students during their study. Computer simulations and computations have the advantage of allowing a student to make judgments, and also to make errors. The process of interactive learning through testing, evaluation, decision-making, and error correction creates a much stronger learning environment than passive instructions. It also improves students' learning activities helping them to reach with success their university degree.

The paper extends the use and explores new possibilities of the Maxima software (GNU Computer Algebra System).

\section{MAXIMA SOLUTION}

Maxima is the software environment that enables to accomplish various mathematical operations including symbolic calculations. It presents a computer algebra system that is oriented to symbolic solutions of given problems. In this way it can be an alternative for such programs as Maple, Mathematica or Matlab with Symbolic Toolbox are.

The disadvantage of Maxima can lie in the fact that it has not so many toolboxes for so many areas as mentioned proprietary software environments. In the paper we pre- sent a created Maxima support for selected topics in Automation area.

Our attention was devoted mainly to the solution of tasks via Internet. Since Maxima is the software that is determined for the installation on a local computer it was necessary to find a way how to explore it online. To transform Maxima to an online tool it was necessary to build an interface that would enable to approach the program kernel via Internet. Actually, we decided to implement two interfaces:

- graphical user interface that offers users to use all Maxima functions via the command line available on the web page [15].

- program interface that enables to exploit the Maxima functionality in other web applications.

The first possibility enables to put Maxima software on the server and to allow students to use it via command line (or command area) for mathematical computations without need to take care about the installation on own computer.

The second possibility enables to exploit the Maxima functionality for building various external web applications. In the paper we introduce several applications that can be used for teaching Control Theory that is one of the fundamental subjects that are taught at universities of technical orientation.

\section{MaXima Online ToOl Technology}

The functionality of the created Maxima based online tool is sketched in Fig.1.

All commands that have to be processed by Maxima are sent from an external web application to the server side PHP script. The script validates the data (from the user or from an external application) and according to their type it decides about their next manipulation. Actually, the script can receive two different inputs:

- simple command that can be forwarded for computation to Maxima software without any need for additional command processing.



Figure 1. Schematic block scheme of Maxima online implementation 
- graph plotting command - in this case the script needs to generate the filename for the image file which is going to carry the image of the plotted graph.

However, in both situations we need to transfer commands received from the external application to the form that is acceptable by Maxima. Therefore it is necessary to create a batch string containing Maxima commands that can be executed via shell_exec() PHP function whereby the system shell enables to start the installed version of Maxima.

After Maxima gets and executes the batch string, the Maxima output is sent back to the shell as the text string and the shell forwards it to the PHP script. In the case that the batch string contained graph plotting commands Maxima generates the necessary output and call Gnuplot application to generate PNG image file from Maxima output with the already generated filename.

The PHP script receives the result of Maxima computation as the string from the shell_exec() function. The script transforms the Maxima output to the TeX format. Finally, the web page with the Maxima output can be generated and visualized in the web browser to the interested user.

An external web application can use two formats for visualization of mathematical results (JSMath or PNG images). If the mathematical results should be displayed by JSMath tool the output is generated with the TeX representation of received strings and JSMath will take care about all the remaining job. Otherwise the PNG images of results are generated using MATHTEX and DVIPNG tools.

Since Maxima software is used as an engine for executing all required computations in an external web application, the mathematical tasks for Maxima are indicated not by the user but by the other application. Similarly, the results from Maxima are sent not to the user but they are sent back to the online application that created the operation request. Actually, the user doesn't need to know about the existence of Maxima environment on the server. The created program interface includes methods that enable interaction between Maxima and a new web application.

\section{DEVELOPED WEB APPLICATIONS}

The Control Theory is one of the fundamental subjects that are taught at universities of technical orientation. At the Faculty of Electrical Engineering and Information Technology (Slovak University of Technology) in Bratislava it is placed into the second year of the study.

The course is considered as an introduction to analysis and design of feedback control systems. It presents an integrated approach to control theory both from the frequency domain and time domain. The big emphasis is devoted to modeling of various systems. Among other topics you can find stability of systems; root locus, Bode plots and Nyquist criterion; controllability and observability; pole placement, etc. In created applications we tried to illustrate in an interactive way at least some of these topics.

Nowadays the front end of the web application can be created by various technologies. The developer can use Java, Flash, JavaScript in combination with DOM and $\mathrm{CSS}$, etc. Each of them brings its pros and cons. After consideration several factors in this paper we decided to

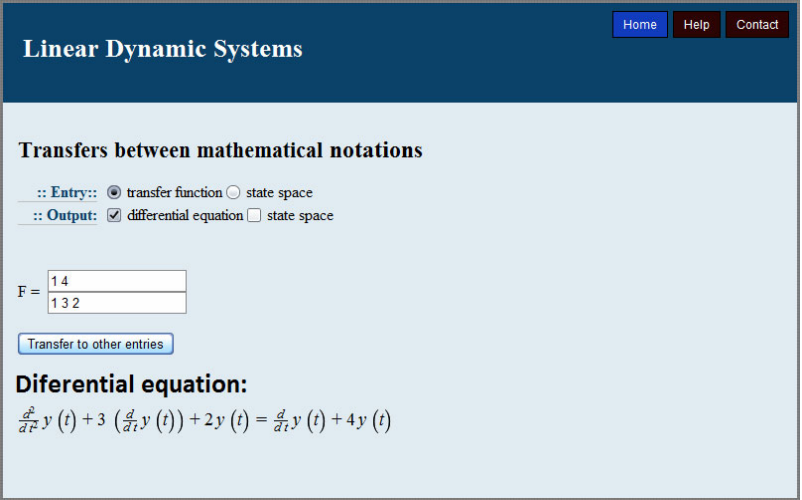

Figure 2. Front-end of the application for model conversion

combine classical HTML with CSS and JavaScript technology. It enables to create well-looking output in a quite simple way. The graphical user interface is supported by other complementary technologies such as JQuery, JSMath and Yetii.

\section{A. Transformation of Mathematical Model Description}

For the control design it is really important to find the exact possible mathematical model of a system. Mathematical models can be described by different forms. Depending on the particular system and the particular circumstances, one mathematical model can be better than another one. For example, in optimal control problems, it is advantageous to use state-space representations. On the other hand, for the transient response or frequency response analysis of single input single output, linear, timeinvariant systems, the transfer function representation may be more convenient than any other [5]. Another possibility is to use the differential equation description. Once a mathematical model of a system is obtained, various analytical and computer tools can be used for analysis and synthesis purposes.

Fig.2 illustrates the developed application that enables to transform one form of the dynamical system description to the other one. The user can enter the transfer function of the system or its state space representation and as a result he or she can get the system differential equation or the state space representation in a selected canonical form. This application helps students to understand and to practice all types of system transformations that they should learn.

\section{B. RLC Circuit Solution}

Another application enables modeling of RLC circuits. The client application (see Fig. 3) allows to build RLC circuits by simple drag and drop procedure. The circuit structure can contain one input voltage and arbitrary number of passive components such as resistor, inductor and capacitor. By default the output of the system is determined by the voltage on the last entered element. However, it can be easily changed. The circuit structure can also be saved in an intermediate stage so the user is not obliged to finish the whole problem solution in one session. He or she can return to it later.

Using this application the user can find mathematical model of the created circuit, i.e. mathematical dependence between input and output voltage.

After defining the RLC circuit structure the incidence matrix and the vector of RLC components is generated. It 


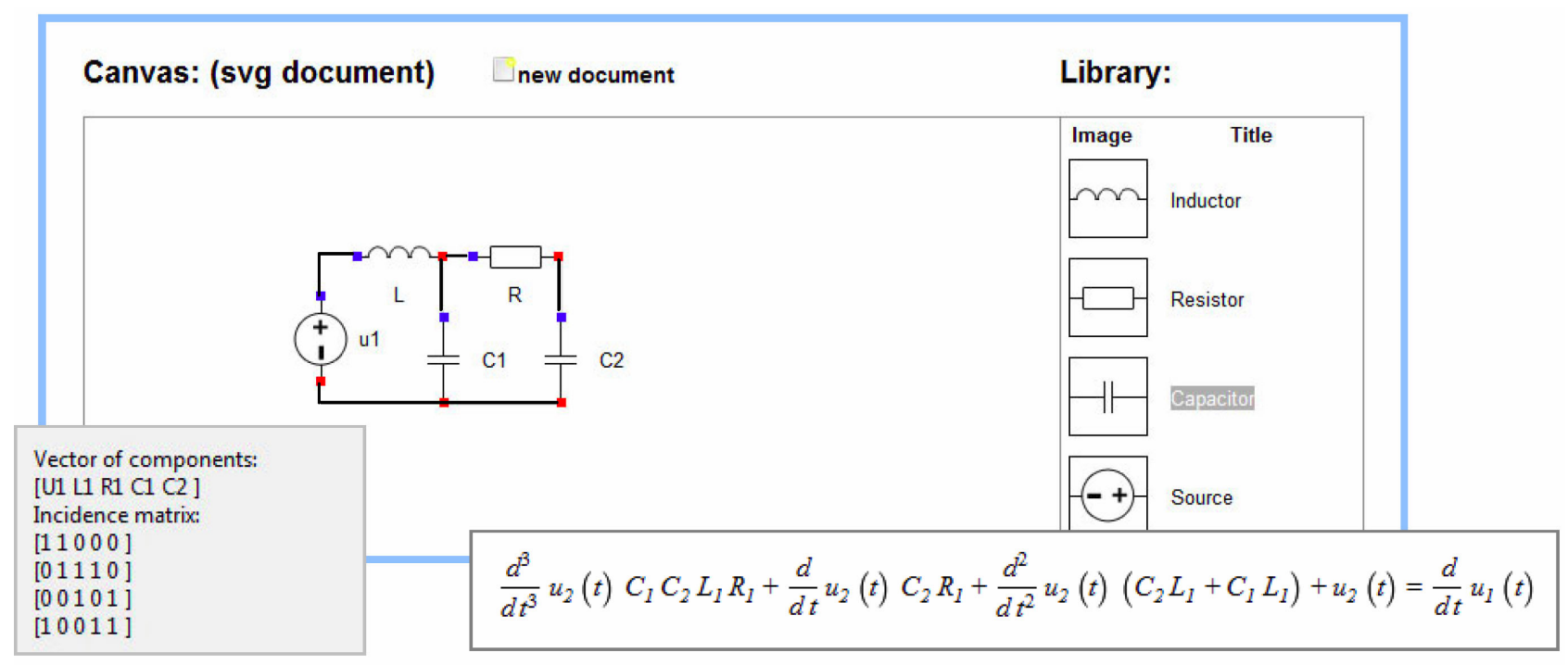

Figure 3. Web application for modeling of RLC circuits

is done automatically by the server script on the base of position of all elements in the structure. Then, the matrix and the vector are sent to Maxima environment where differential equation, transfer function or/and state space representation of the model are calculated. The results are sent back to the server script that enables their visualization for the user.

Of course, the created Maxima procedure can also be used independently from the presented web application. In such a case, the user has to enter the incidence matrix and the vector of RLC circuit components in the Maxima environment manually. Then, these variables can be used as input arguments for the developed procedure.

The advantage of the online solution consists in the fact that students are not forced to use Maxima directly, it is incorporated in the kernel of application, and therefore there is no need to learn Maxima syntax for those who are not interested in it.

Our aim is to extend this application in such a way that it would serve not only for solution of RLC circuits but also for solution of mechanical systems where instead of resistor, inductor and capacitor we will consider mass, spring and damper.

\section{Block Scheme Simplification}

The next developed application is the application that enables simplification of the block scheme. The client application (see Fig. 4) enables to build a general linear SISO circuit again by simple drag and drop procedure. The control structure can contain input, output, sum elements and several blocks that can be described by the transfer function defined either in numerical or in analytical way.

After defining the block scheme user can find the resulting transfer function that describe the behavior of the whole system. The background computation is done in Maxima. It is again necessary to define the system description on the base of position of all elements and all connections in the block scheme structure. The whole problem can be converted to the solution of linear equations. Their number is depending on the number of blocks in the control structure.
However, in spite of many advantages this application together with the previous ones brings one crucial problem. Students receive the whole solution of the task in a very comfortable way. It is sufficient only to draw a circuit or a scheme, to press a button and student has immediately the correct result. It is not necessary to enter neither any parameter. Therefore the applications should serve only as a support tool for checking results. They are not able to teach student all steps of the solution.

\section{Building of Next Applications}

In spite of the fact that we introduced here only three applications it is not the end of our efforts. Our aim is to continue in this direction and to build set of applications that will support the whole course of the subject Control Theory. In present time we are building applications for demonstration of characteristics of linear systems, i.e. transient response, impulse response and frequency characteristics. Then, we would like to devote our attention to the stability of systems and to other basic topics. Advantage is that all computations can be done in Maxima environment and we take care only for front end of application, communication with back end and interaction with student.

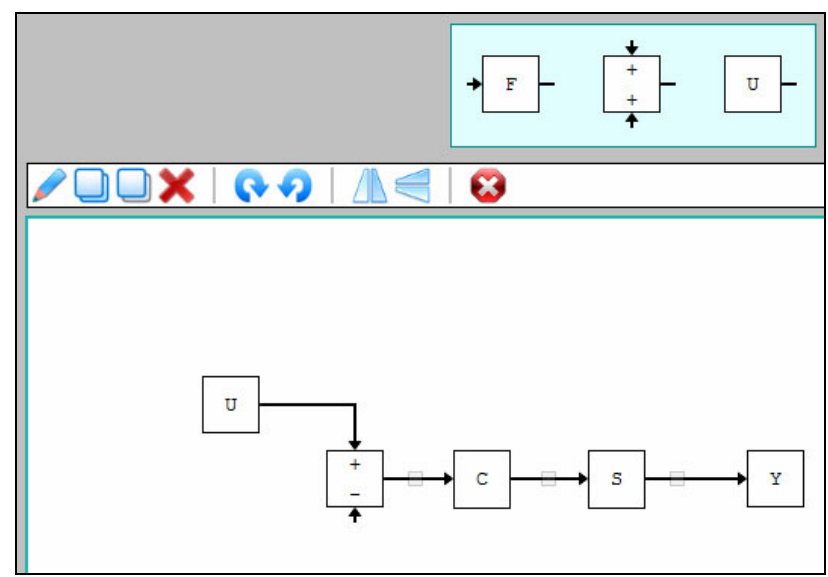

Figure 4. Web application for simplification of block scheme 


\section{CONCLUSIONS}

The paper presented a tool for developing of online applications oriented to the interactive solutions of examples in Control Theory. We exploited a possibility to use Maxima software for doing all computations.

The advantage is that the tool is available online via Internet for all interested users in any time they need. Since the presented topic is part of the study at probably all technical universities devoted to electrical engineering it presents the useful instrument in an educational process mainly in online and distance forms of education.

The last thing to mention is the fact that each version of the introduced application was prepared by regular students in frame of their individual projects. Actually, for example the front end of the application for solution of RLC circuits represents already the 4th solution of the graphical design. In frame of the projects students tried to apply Flash technology, Java applets and Javascript. Step by step the solutions are better and better. Of course, applications could also be done by professional programmer, but we decided to give opportunity to students. They like programming and together with it they learn control theory, too. The idea of "learning by doing" is in this case very actual and the results showed that it is good to continue in this direction also in future.

\section{ACKNOWLEDGMENT}

Author thanks to Dávid Gyurász, Ludovít Vörős, Peter Beňo and Zoltán Magyar for their help with the problem algorithmization and programming.

\section{REFERENCES}

[1] B. Gaertner, Maxima Programming, 2005. http://maxima.source forge.net/docs/tutorial/en/gaertner-tutorial-revision/Pages/Program ming0001.htm

[2] C. P. Leão, A. E. Rodrigues: Transient and steady-state models for simulated moving bed processes: numerical solutions. Computers \& Chemical Engineering 28(9): 1725-1741, 2004.

[3] P. Lutus, Symbolic Mathematics Using Maxima, http://arachnoid.com/maxima/

[4] Z. Magyar, T. Starý, L. Szolik, L'. Vörös, K. Žáková, "Modeling of Linear Dynamical Systems Using Open Tools", 10th international Conference "Virtual University", Bratislava, Slovak Republic, December 2009.
[5] K. Ogata, Modern Control Engineering, 3rd Edition, Prentice Hall London, 1997.

[6] J. Resig and the jQuery Team. 2009. jQuery Documentation. http://docs.jquery.com

[7] M. T. Restivo, J. Mendes, A.M. Lopes, C.M. Silva, F. Chouzal, A Remote Lab in Engineering Measurement, IEEE Trans. on Industrial Electronics, vol. 56, no.12, pp. 4436-4843, 2009.

[8] F. Schauer, M. Ožvoldová, F. Lustig, „Real Remote Physics Experiments across Internet - Inherent Part of Integrated ELearning", Int. Journal of Online Engineering (iJOE), 4, No 2, 2008.

[9] Chr. Schmid, „Internet - basiertes Lernen“, Automatisierungstechnik, 51, No. 11, p. 485-493, 2003.

[10] M. Šimunek, P., Bisták, M. Huba, „Virtual Laboratory for Control of Real Systems", Conference Proceedings ICETA, Košice, Slovakia, 2005

[11] A. J. Turgeon, "Implications of Web-Based Technology for Engaging Students in a Learning Society", Journal of Public Service and Outreach 2(2): 32-37.

[12] E. L. Woollett, Maxima by example, http://www.csulb.edu/ $\sim$ woollett/

[13] K. Žáková, "Control Theory - an interactive online course", 6th international conference "Virtual University", Bratislava, December, 2005.

[14] K. Žáková, M. Sedlák, "Remote Control of Experiments via Matlab", Int. Journal of Online Engineering (iJOE), 2, No. 3, 2006.

[15] K. Žáková, ”A Tool for Support of Online Symbolic Computations", Int. Conference "Cybernetics and Informatics", Vyšná Boca, Slovak Republic, February 2010.

[16] K. Žáková, Z. Magyar, L.Vörös, “Online Modeling of RLC Circuits”, Remote Engineering \& Virtual Instrumentation Conference REV'2010, Stockholm, Sweden, 2010.

[17] jsMath: A Method of Including Mathematics in Web Pages, http://www.math.union.edu/ dpvc/jsMath/, Verified: 10 Mar 2009.

\section{AUTHOR}

K. Žáková is with the Faculty of Electrical Engineering and Information Technology, Slovak University of Technology, Ilkovičova 3, 81219 Bratislava, Slovakia (e-mail: katarina.zakova@stuba.sk).

The work has been partially supported by the Grant KEGA No. 3/7245/09 and by the Grant VEGA No. 1/0656/09. This support is very gratefully acknowledged.

Submitted, March $15^{\text {th }}, 2011$. Published as resubmitted by the authors April $20^{\text {th }}, 2011$. 